Research Paper

\title{
Impact of operation duration on short-term and long-term prognosis in patients undergoing radical colorectal surgery
}

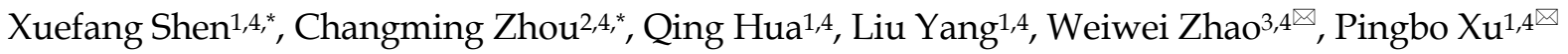 \\ 1. Department of Anesthesiology, Fudan University Shanghai Cancer Center, Shanghai, China. \\ 2. Department of Cancer Prevention, Fudan University Shanghai Cancer Center, Shanghai, China. \\ 3. Department of Integrated Therapy, Fudan University Shanghai Cancer Center, Shanghai, China. \\ 4. Department of Oncology, Shanghai Medical College, Fudan University, Shanghai, China. \\ ${ }^{*}$ Co-first authors with equal contributions to this work. \\ $\triangle$ Corresponding authors: Pingbo Xu, Department of Anesthesiology, Fudan University Shanghai Cancer Center, Shanghai, China; E-mail: \\ xupingboshanghai@163.com; and Weiwei Zhao, Department of Integrated Therapy, Fudan University Shanghai Cancer Center, Shanghai, China; E-mail: \\ sunbrightzww@126.com.
}

(1) The author(s). This is an open access article distributed under the terms of the Creative Commons Attribution License (https://creativecommons.org/licenses/by/4.0/). See http://ivyspring.com/terms for full terms and conditions.

Received: 2021.08.07; Accepted: 2021.12.11; Published: 2022.01.16

\begin{abstract}
Purpose: The objective of this study was to evaluate the impact of operation duration on short- and long-term outcomes of colorectal cancer patients following surgical resection.

Methods: 6224 consecutive patients who underwent radical colorectal surgery were retrospectively assessed and were divided into short operation duration group (SOD) and long operation duration group (LOD) according to the operation duration cutoff value of 110 minutes.

Results: Compared with patients in LOD group, patients in SOD group had significantly lower total costs in hospital, reduced expenses for drugs and antibiotics, shorter length of stay (LOS) in hospital and in the ICU. Moreover, 5-year overall survival (OS) and disease-free survival (DFS) for patients in the SOD group were markedly higher than for patients in the LOD group. Mutivariate regression analysis indicated that longer operation duration was associated with poor prognosis, with a hazard ratio of 1.004 (1.003, $1.005)$ for OS and $1.005(1.003,1.006)$ for DFS. Finally, surgeons' qualifications had meaningful correlation with operation duration $(r=0.450)$.

Conclusions: Operation duration is an independent risk factor for patients' short-term and long-term outcome after radical colorectal surgery. Improve the surgical skills of the surgeon may shorten the operation duration, and further improve the outcome for patients.
\end{abstract}

Key words: operation duration; short-term and long-term prognosis; colorectal cancer; radical surgery

\section{Introduction}

According to global cancer statistics for 2018, colorectal cancer (CRC) was the fourth most commonly diagnosed cancer and the second highest cause of cancer-related death [1]. Radical surgical resection is currently the most common approach for colorectal cancer treatment [2]. Optimizing surgical processes may affect postoperative prognosis, which is consistent with the notion of enhanced recovery after surgery (ERAS).

Patient characteristics, such as pathologic
Tumor-Node-Metastasis (pTNM), poor differentiated clusters of neoplastic cells [3], and tumor location [4] are important factors that affect outcomes. However, operation duration is one of the key operational factors. Growing bodies of studies suggest that long operation duration is associated with poor perioperative prognosis, such as venous thromboembolism (VTE) [5], infection [6], and even overall rate of perioperative complications [7].

Although there have been many studies 
concerning the association between operation duration and postoperative complications, there have been no consistent conclusions [6, 8-12]. Some researchers have suggested that prolonged operation duration, especially lasting over 4 hours, can increase postoperative complications and length of stay (LOS) in hospital, and can have a negative impact on the outcome of enhanced recovery after laparoscopic colorectal resection $[6,8,10,11]$. Other studies have suggested that prolonged operation duration does not increase postoperative morbidity [9, 12]. Moreover, only a few studies have focused on the effect of operation duration on long-term prognosis. Wang et al. found that prolonged operation duration was associated with periprosthetic joint infection within 1 year after primary joint arthroplasty [13]. Additionally, there are currently few studies that have reported on the relationship between long-term prognosis and prolonged operation time in patients undergoing tumor resection. Therefore, the current study aimed to explore the effects of operation duration on postoperative short-term and long-term outcomes in a large cohort of patients undergoing radical resection for CRC.

\section{Materials and Methods}

\section{Data collection}

Between July 1, 2011 and December 31, 2016, all consecutive patients who underwent radical resection for colorectal cancer at Fudan University Shanghai Cancer Center (FUSCC) were recruited for this retrospective study. The inclusion criteria were as follows: (1) age >18 years; (2) standard open or laparoscopic radical resection of colorectal cancer confirmed by pathology; (3) local lesions without distant metastasis; (4) availability of all clinical data. Patients with metastasis, recurrence, or other palliative surgery were excluded. In addition, patients who underwent operations by surgeons with less than 10 years' surgical experience were excluded to guarantee the quality of the operation. From the database of the FUSCC clinical information system, medical information, demographic information, tumor location, pathological diagnosis, pathological stage, operation methods, extramural vascular invasion (EMVI), perineural invasion (PNI), circumferential radial margin (CRM), anesthesia methods, ASA class, routine blood tests, medical costs, and number of lymph nodes examined were extracted. The primary endpoint was the long-term prognosis for patients, including overall survival (OS) and disease-free survival (DFS). The secondary endpoint was the short-term prognosis, including costs, LOS in hospital, and LOS in the ICU. The last follow-up date was November 30, 2019. The study was approved by the institutional review committee of FUSCC (Shanghai, China). Informed consent was waived due to the retrospective nature of the study.

\section{Statistical methods}

Mean \pm standard deviation (SD) and median (q1, q3) were used to describe continuous variables, while frequencies and percentages were used for categorical variables. Chi-square test or Fisher's exact test (as appropriate) were applied when testing the variables' differences between different operation duration groups for categorical variables; Wilcoxon sum rank test or Kruskal-Wallis $\mathrm{H}$ test were applied when testing differences between different operation duration groups for continuous variables. The Kaplan-Meier method was used to calculate survival rate, and log-rank test was used to compare differences between the groups. Cox proportional hazards models were used to calculate the univariate and multivariate adjusted risk ratios (HRs) and 95\% confidence intervals (CIs). Univariate and multivariate analyses were performed for all significant variables, except operation time, to identify survival-confounding factors. Then, propensity score matching (PSM) was utilized between groups to reduce the influence of these confounding factors. PSM was conducted in R ( $R$ Core Team, 2014) and the rest of the statistical analyses were performed using IBM SPSS Statistics for Windows, version 25.0 (IBM Corp., Armonk, N.Y., USA).

\section{Results}

\section{Patient characteristics}

Between 2011 and 2016, 9135 patients aged >18 years underwent colorectal resection, and their records were retrieved from the database. 879 patients with metastasis, recurrence, or palliative surgery, were excluded from them. Then, 2032 patients undergoing surgery by surgeons with less than 10 years' surgical experience were excluded to eliminate the influence of surgeons' experience on prognosis. Finally, 6224 patients with colorectal cancer were eligible for analysis. The patient attrition flow chart is shown in Supplementary Fig. 1.

The histogram of CRC patients' operative duration is shown in Supplementary Fig. 2. The levels of operative duration had positively skewed distributions. The mean $( \pm S D)$ and median $(q 2, q 3)$ of operation duration were $111.00 \pm 41.35$ and 102.00 $(82.00,131.00)$ minutes, respectively, with a minimum of 45 minutes and a maximum of 445 minutes. In order to facilitate the analysis of the relationship 
between operative duration and clinical outcomes, 110 minutes of operative duration was selected as the grouping criterion. Patients were then assigned into the SOD group $(\leq 110 \mathrm{~min}, \mathrm{n}=3668)$ or LOD group ( $>$ $110 \mathrm{~min}, \mathrm{n}=2556$ ).

The clinico-pathological characteristics of the study population according to the two groups are given in Table 1. Preoperative hemoglobin, platelet, serum creatinine and albumin levels, pTNM staging, EMVI+, PNI+, and number of lymph nodes examined were similar between the groups $(P>0.05)$. However, there was a greater proportion of female patients, older adults ( $\geq 65$ years), overweight and obesity patients, laparoscopic surgery, carcinoma of the rectum (vs right or left colon cancer), signet-ring cell carcinoma (vs adenocarcinoma or mucinous adenocarcinoma), CRM+, combined anesthesia $(\mathrm{GA}+\mathrm{EA})$, and ASA III-V (vs I or II) in the LOD group compared with the SOD group $(P<0.05)$. Then, the CRC patients in the SOD group and LOD group were separately matched $(1: 1)$ by PSM. The confounding factors, such as gender, age, surgical approach, pTNM staging, tumor site, histological type, EMVI, PNI, CRM, number of lymph nodes examined, hemoglobin, and albumin were well matched in the two cohorts, and none was found to differ significantly between the pairs at baseline (Supplementary Table 1 and Supplementary Fig. 3).

Table 1. Characteristics of patients with different operation duration

\begin{tabular}{|c|c|c|c|c|c|c|c|c|}
\hline & & $\leq 110 \mathrm{~min}$ & & $>110 \mathrm{~min}$ & & & & \\
\hline Characteristics & & $\mathrm{N}(\%)$ & Mean $( \pm S D)$ & $\mathrm{N}(\%)$ & Mean $( \pm S D)$ & Chi2 & $t$ & P value \\
\hline Gender & Male & $2127(57.99)$ & & $1600(62.60)$ & & 13.32 & & $<0.001^{*}$ \\
\hline & Female & $1541(42.01)$ & & $956(37.40)$ & & & & \\
\hline Age Group (year) & $<45$ & $399(10.88)$ & & $334(13.07)$ & & 18.47 & & $0.001^{*}$ \\
\hline & $45-54$ & $685(18.68)$ & & $510(19.95)$ & & & & \\
\hline & $55-64$ & $1271(34.65)$ & & $900(35.21)$ & & & & \\
\hline & $65-74$ & $903(24.62)$ & & $591(23.12)$ & & & & \\
\hline & $75+$ & $410(11.18)$ & & $221(8.65)$ & & & & \\
\hline Surgical Approach & Open Surgery & $3488(95.09)$ & & $1577(61.70)$ & & 1108.54 & & $<0.001^{*}$ \\
\hline & Laparoscope & $180(4.91)$ & & $979(38.30)$ & & & & \\
\hline BMI kg/m² & Normal (18.5-23.9) & $2096(61.29)$ & & $1324(38.71)$ & & 30.488 & & $<0.001^{*}$ \\
\hline & Underweight $(<18.5)$ & $236(63.96)$ & & $133(36.04)$ & & & & \\
\hline & Overweight (24-29.9) & $1264(55.37)$ & & $1019(44.63)$ & & & & \\
\hline & Obesity $(\geq 30)$ & $70(48.28)$ & & $75(51.72)$ & & & & \\
\hline pTNM Staging & I & $710(19.36)$ & & $515(20.15)$ & & 0.65 & & 0.722 \\
\hline & II & $1309(35.69)$ & & $910(35.60)$ & & & & \\
\hline & III & $1649(44.96)$ & & $1131(44.25)$ & & & & \\
\hline Tumor Site & Rectum & 1997(55.49) & & $1560(61.47)$ & & 37.48 & & $<0.001^{*}$ \\
\hline & Left Colon & $866(24.06)$ & & $449(17.69)$ & & & & \\
\hline & Right Colon & $736(20.45)$ & & $529(20.84)$ & & & & \\
\hline Histological type & Adenocarcinoma & $3241(88.36)$ & & $2229(87.21)$ & & 7.97 & & $0.019 *$ \\
\hline & Mucinous adenocarcinoma & $377(10.28)$ & & $268(10.49)$ & & & & \\
\hline & Signet-ring cell carcinoma & $50(1.36)$ & & $59(2.31)$ & & & & \\
\hline EMVI & - & $2885(78.65)$ & & $2039(79.77)$ & & 1.14 & & 0.285 \\
\hline & + & $783(21.35)$ & & $517(20.23)$ & & & & \\
\hline PNI & - & $2930(79.88)$ & & 2056(80.44) & & 0.29 & & 0.587 \\
\hline & + & $738(20.12)$ & & $500(19.56)$ & & & & \\
\hline CRM & - & $3643(99.32)$ & & $2519(98.55)$ & & 8.96 & & $0.003^{*}$ \\
\hline & + & $25(0.68)$ & & $37(1.45)$ & & & & \\
\hline Anesthesia & $\mathrm{GA}+\mathrm{EA}$ & $2248(61.29)$ & & $1477(57.79)$ & & 7.68 & & $0.006^{*}$ \\
\hline & GA & $1420(38.71)$ & & $1079(42.21)$ & & & & \\
\hline ASA & I & $1249(34.05)$ & & $871(34.08)$ & & 7.11 & & $0.029^{*}$ \\
\hline & II & $2335(63.66)$ & & $1598(62.52)$ & & & & \\
\hline & III-V & $84(2.29)$ & & $87(3.40)$ & & & & \\
\hline Number of lymph $n$ & xamined & & $16.69 \pm 6.42$ & & $16.64 \pm 7.24$ & & 0.281 & 0.779 \\
\hline Hemoglobin $(\mathrm{g} / \mathrm{l})$ & & & $126.76 \pm 20.30$ & & $125.95 \pm 21.01$ & & 1.502 & 0.133 \\
\hline Number of platelets & & & $230.71 \pm 78.50$ & & $232.41 \pm 80.27$ & & -0.821 & 0.412 \\
\hline Albumin (g/l) & & & $42.05 \pm 3.79$ & & $42.03 \pm 4.00$ & & 0.163 & 0.871 \\
\hline Creatinine $(\mu \mathrm{mol} / \mathrm{l})$ & & & $70.84 \pm 17.84$ & & $71.41 \pm 18.54$ & & -1.185 & 0.236 \\
\hline Alkaline phosphata & & & $73.72 \pm 21.92$ & & $75.23 \pm 28.39$ & & -2.056 & $0.040^{*}$ \\
\hline Total cost (RMB) & & & $43559 \pm 13298$ & & $48769 \pm 17031$ & & -13.531 & $<0.001^{*}$ \\
\hline Length of stay in ho & (days) & & $14.91 \pm 6.64$ & & $16.40 \pm 7.41$ & & -8.341 & $<0.001^{*}$ \\
\hline Length of stay in IC & & & $0.18 \pm 1.17$ & & $0.24 \pm 1.22$ & & -1.990 & $0.047^{*}$ \\
\hline Cost of drug (RMB) & & & $15530 \pm 8130$ & & $17270 \pm 9779$ & & -7.637 & $<0.001^{*}$ \\
\hline Cost of antibiotics ( & & & $940 \pm 1158$ & & $1080 \pm 1377$ & & -4.362 & $<0.001^{*}$ \\
\hline Total & & $3668(58.93)$ & & $2556(41.07)$ & & & & \\
\hline
\end{tabular}




\section{Longer operation duration was related to poor prognosis for CRC patients}

As shown in Table 1, patients in the SOD group vs the LOD group had lower total costs in hospital $(43558 \pm 13298 \mathrm{CNY}$ vs $48768 \pm 17031 \mathrm{CNY}$, respectively; $P<0.001)$, lower drug costs $(15529 \pm 8129$ CNY vs $17269 \pm 9778 \mathrm{CNY} ; P<0.001)$ and lower antibiotic costs $(939 \pm 1158 \mathrm{CNY}$ vs. $1080 \pm 1377 \mathrm{CNY}$, $P<0.001)$, shorter LOS in hospital (14.91 \pm 6.64 days vs $16.40 \pm 7.41$ days; $P<0.001$ ) and shorter LOS in the ICU $(0.18 \pm 1.17$ days vs $0.24 \pm 1.22$ days; $P=0.047)$. On univariate COX regression analysis, prolonged operation duration was found to be associated with higher total costs in hospital (especially the cost of drugs and antibiotics) and longer LOS in hospital and LOS in the ICU (Table 2).

Table 2. Univariate regression of operation duration on patients' short-term effect

\begin{tabular}{llll}
\hline & $\beta$ & $\mathrm{t}$ & $\mathrm{P}$ value \\
\hline \multirow{2}{*}{$\begin{array}{l}\text { Constant } \\
\text { operation duration }\end{array}$} & 36323.520 & 67.800 & $<0.001^{*}$ \\
& 84.463 & 18.674 & $<0.001^{*}$ \\
Constant & Inpatient days (days) & & \\
operation duration & 12.806 & 50.879 & $<0.001^{*}$ \\
& 0.024 & 11.509 & $<0.001^{*}$ \\
Constant & Drug Fees (RMB) & & \\
operation duration & 12704.484 & 39.820 & $<0.001^{*}$ \\
& 31.892 & 11.840 & $<0.001^{*}$ \\
Constant & Antibiotic fees (RMB) & & \\
operation duration & 708.937 & 15.620 & $<0.001^{*}$ \\
\hline
\end{tabular}

During a mean longitudinal follow-up period of 56.0 months (95\% CI 55.2-56.9) after surgery, 1117 deaths occurred (17.9\%) and 1515 patients $(24.3 \%)$ had recurrences. The Kaplan-Meier cumulative survival curves for OS and DFS for patients in the two groups are shown in Fig. 1. The 5-year OS for patients was $82.0 \%$ in the SOD group and $76.7 \%$ in the LOD group

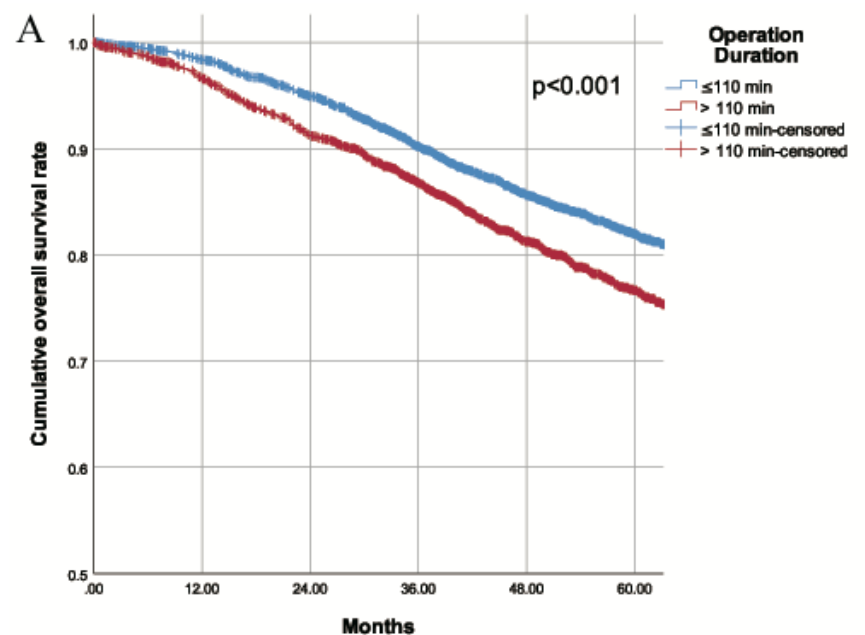

$(P<0.001)$. Similarly, the 5 -year DFS was $76.2 \%$ in the SOD group and $68.7 \%$ in the LOD group $(P<0.001)$. Furthermore, the OS and DFS were compared between the two matched cohorts after PSM. Likewise, obviously worse OS and DFS were observed in the LOD group in comparison with PSM controls (Supplementary Fig. 4). These results revealed that shorter operation duration was associated with higher OS and DFS in CRC patients undergoing colorectal resection. With each additional minute prolonging surgery, the risk of death increased by $0.4 \%$ and risk of recurrence/metastasis/ death increased by $0.5 \%$. Although the unit effect was quite small, the risk increased by $24 \%$ when the surgery was prolonged by one hour.

\section{Factors related to long-term prognosis}

Univariate and multivariate Cox proportional hazards analyses were performed to evaluate the relationship between operation duration and long-term prognosis (OS and DFS) (Tables 3 and 4). On univariate COX regression, longer operation duration was found to be associated with poor prognosis, with a HR of $1.004(1.002,1.005)$ for OS and $1.004(1.003,1.005)$ for DFS. After adjusting for confounders such as sex, age, pTNM staging, tumor site, histological type, EMVI, PNI, CRM, blood haemoglobin and serum albumin level, number of lymph nodes examined, and surgical approach, operation duration was found to be an independent risk factor for OS with a HR of $1.004(1.003,1.005)$, and a HR of $1.005(1.003,1.006)$ for DFS. In addition to operation duration, gender, age, pTNM, tumor site, histological type, EMVI, PNI, CRM, hemoglobin, albumin, and number of lymph nodes extracted were all significantly associated with OS, and with DFS $(\mathrm{P}<0.001)$.

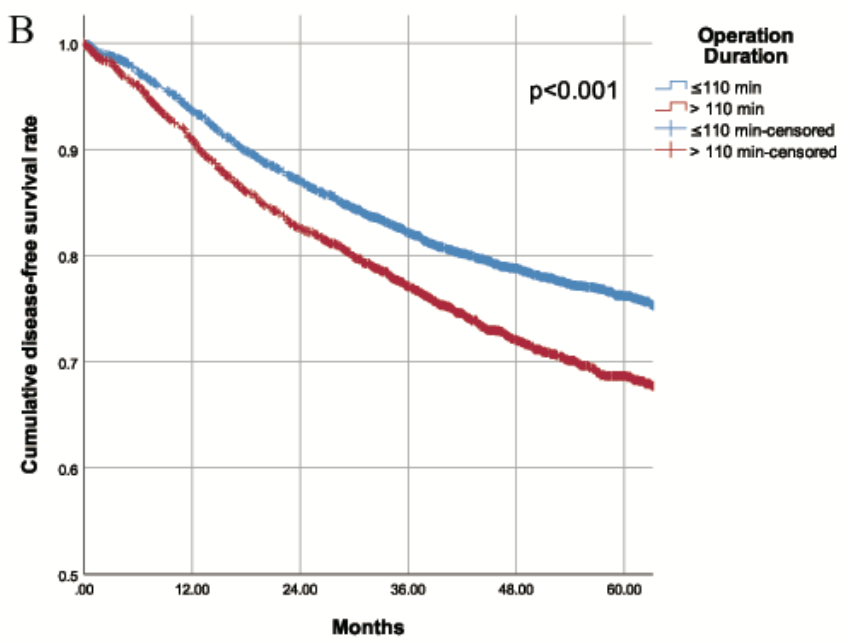

Figure 1. A. Overall survival of patients with different operation duration B. Disease free survival of patients with different operation duration 
Table 3. Univariate and multivariate regression of factors affecting OS using Cox regression

\begin{tabular}{|c|c|c|c|c|c|c|}
\hline \multirow[t]{2}{*}{ Characteristics } & \multicolumn{3}{|c|}{ Univariate } & \multicolumn{3}{|c|}{ Multivariate } \\
\hline & $\beta$ & $P$ value & HR (95\% CI) & $\beta$ & $P$ value & $\mathrm{HR}(95 \% \mathrm{CI})$ \\
\hline Female/Male & -0.103 & 0.095 & $0.902(0.800,1.018)$ & -0.213 & $0.002^{*}$ & $0.808(0.706,0.924)$ \\
\hline Age Group & & $<0.001^{*}$ & & & $<0.001^{*}$ & \\
\hline $45-54 /<45$ & -0.003 & 0.979 & $0.997(0.785,1.266)$ & 0.213 & 0.103 & $1.237(0.958,1.598)$ \\
\hline $55-64 /<45$ & 0.039 & 0.727 & $1.039(0.837,1.290)$ & 0.288 & $0.016^{*}$ & $1.334(1.055,1.686)$ \\
\hline $65-74 /<45$ & 0.274 & $0.015^{*}$ & $1.315(1.054,1.641)$ & 0.395 & $0.002^{*}$ & $1.484(1.162,1.896)$ \\
\hline $75+/<45$ & 0.959 & $<0.001^{*}$ & $2.610(2.074,3.283)$ & 1.076 & $<0.001^{*}$ & $2.932(2.265,3.795)$ \\
\hline Laparoscope/Open Surgery & 0.002 & 0.977 & $1.002(0.854,1.176)$ & & & \\
\hline BMI & & $0.007^{*}$ & & & & \\
\hline Underweight/Normal & 0.300 & $0.010^{*}$ & $1.349(1.075,1.693)$ & & & \\
\hline Overweight/Normal & -0.058 & 0.366 & $0.943(0.831,1.070)$ & & & \\
\hline Obesity/Normal & -0.403 & 0.084 & $0.668(0.423,1.055)$ & & & \\
\hline pTNM & & $<0.001^{*}$ & & & $<0.001^{*}$ & \\
\hline II/I & 0.528 & $<0.001^{*}$ & $1.695(1.343,2.140)$ & 0.420 & $0.001^{*}$ & $1.522(1.189,1.947)$ \\
\hline III/I & 1.368 & $<0.001^{*}$ & $3.927(3.171,4.862)$ & 1.030 & $<0.001^{*}$ & $2.801(2.217,3.538)$ \\
\hline Site & & $<0.001^{*}$ & & & $0.007^{*}$ & \\
\hline Left Colon/Rectum & -0.159 & $0.001^{*}$ & $0.853(0.730,0.997)$ & -0.257 & $0.002^{*}$ & $0.773(0.656,0.911)$ \\
\hline Right Colon/Rectum & -0.010 & $0.002^{*}$ & $0.990(0.853,1.149)$ & -0.137 & 0.121 & $0.872(0.734,1.037)$ \\
\hline histological type & & $<0.001^{*}$ & & & $<0.001^{*}$ & \\
\hline mucinous adenocarcinoma / adinocacinoma & 0.299 & $0.001^{*}$ & $1.349(1.131,1.609)$ & 0.281 & $0.003^{*}$ & $1.325(1.098,1.598)$ \\
\hline Signet-ring cell carcinoma / adinocacinoma & 1.138 & $<0.001^{*}$ & $3.121(2.314,4.211)$ & 0.615 & $<0.001^{*}$ & $1.850(1.326,2.583)$ \\
\hline EMVI+/- & 0.968 & $<0.001^{*}$ & $2.634(2.331,2.975)$ & 0.573 & $<0.001^{*}$ & $1.773(1.539,2.043)$ \\
\hline $\mathrm{PNI}+/-$ & 0.860 & $<0.001^{*}$ & $2.363(2.086,2.677)$ & 0.479 & $<0.001^{*}$ & $1.615(1.405,1.856)$ \\
\hline $\mathrm{CRM}+/-$ & 1.557 & $<0.001^{*}$ & $4.747(3.389,6.648)$ & 1.180 & $<0.001^{*}$ & $3.254(2.281,4.641)$ \\
\hline $\mathrm{GA}+\mathrm{EA} / \mathrm{GA}$ & -0.020 & 0.755 & $0.980(0.866,1.110)$ & & & \\
\hline ASA & & $<0.001^{*}$ & & & & \\
\hline II/I & 0.212 & $0.001^{*}$ & $1.236(1.086,1.406)$ & & & \\
\hline $\mathrm{III}-\mathrm{V} / \mathrm{I}$ & 0.728 & $<0.001^{*}$ & $2.071(1.517,2.828)$ & & & \\
\hline Haemoglobin $(\mathrm{g} / \mathrm{l})$ & -0.009 & $<0.001^{*}$ & $0.991(0.988,0.993)$ & -0.008 & $<0.001^{*}$ & $0.992(0.988,0.996)$ \\
\hline Albumin (g/l) & -0.070 & $<0.001^{*}$ & $0.933(0.919,0.947)$ & -0.042 & $<0.001^{*}$ & $0.959(0.941,0.976)$ \\
\hline Operation duration & 0.004 & $<0.001^{*}$ & $1.004(1.002,1.005)$ & 0.004 & $<0.001^{*}$ & $1.004(1.003,1.005)$ \\
\hline Number of lymph nodes examined & -0.030 & $<0.001^{*}$ & $0.971(0.961,0.980)$ & -0.037 & $<0.001^{*}$ & $0.964(0.953,0.974)$ \\
\hline
\end{tabular}

Table 4. Univariate and multivariate regression of factors affecting DFS using Cox regression

\begin{tabular}{|c|c|c|c|c|c|c|}
\hline \multirow[t]{2}{*}{ Characteristics } & \multicolumn{3}{|c|}{ Univariate } & \multicolumn{3}{|c|}{ Multivariate } \\
\hline & $\beta$ & $\mathrm{P}$ value & $\mathrm{HR}(95 \% \mathrm{CI})$ & $\beta$ & P value & $\mathrm{HR}(95 \% \mathrm{CI})$ \\
\hline Female/Male & 0.002 & 0.977 & $0.918(0.828,1.019)$ & -0.173 & $0.003^{*}$ & $0.841(0.749,0.945)$ \\
\hline Age Group & & $<0.001^{*}$ & & & $<0.001^{*}$ & \\
\hline $45-54 /<45$ & -0.035 & 0.726 & $0.966(0.796,1.173)$ & 0.148 & 0.163 & $1.159(0.942,1.427)$ \\
\hline $55-64 /<45$ & -0.021 & 0.813 & $0.979(0.821,1.167)$ & 0.199 & $0.041^{*}$ & $1.220(1.008,1.476)$ \\
\hline $65-74 /<45$ & 0.071 & 0.448 & $1.074(0.894,1.290)$ & 0.209 & $0.043^{*}$ & $1.232(1.007,1.508)$ \\
\hline $75+/<45$ & 0.558 & $<0.001^{*}$ & $1.748(1.435,2.129)$ & 0.686 & $<0.001^{*}$ & $1.985(1.590,2.478)$ \\
\hline Laparoscope/ Open Surgery & 0.002 & 0.977 & $1.002(0.877,1.145)$ & -0.165 & $0.042^{*}$ & $0.848(0.724,0.994)$ \\
\hline BMI & & $0.011^{*}$ & & & & \\
\hline Underweight/Normal & 0.191 & 0.063 & $1.210(0.990,1.480)$ & & & \\
\hline Overweight/Normal & -0.111 & $0.045^{*}$ & $0.895(0.803,0.998)$ & & & \\
\hline Obesity/Normal & -0.262 & 0.158 & $0.769(0.535,1.107)$ & & & \\
\hline pTNM & & $<0.001^{*}$ & & & $<0.001^{*}$ & \\
\hline II/I & 0.458 & $<0.001^{*}$ & $1.582(1.310,1.909)$ & 0.411 & $<0.001^{*}$ & $1.509(1.235,1.843)$ \\
\hline III/I & 1.205 & $<0.001^{*}$ & $3.336(2.805,3.966)$ & 0.904 & $<0.001^{*}$ & $2.470(2.040,2.990)$ \\
\hline Site & & $0.018^{*}$ & & & $0.005^{*}$ & \\
\hline Left Colon/Rectum & -0.195 & $0.005^{*}$ & $0.823(0.719,0.942)$ & -0.236 & $0.001^{*}$ & $0.790(0.685,0.911)$ \\
\hline Right Colon/Rectum & -0.039 & 0.550 & $0.961(0.845,1.093)$ & -0.098 & 0.199 & $0.906(0.780,1.053)$ \\
\hline histological type & & $<0.001^{*}$ & & & $0.011^{*}$ & \\
\hline mucinous adenocarcinoma / adinocacinoma & 0.199 & $0.013^{*}$ & $1.220(1.044,1.426)$ & 0.175142 & $0.039^{*}$ & 1.191(1.009,1.407) \\
\hline Signet-ring cell carcinoma / adinocacinoma & 0.917 & $<0.001^{*}$ & $2.501(1.905,3.284)$ & 0.356917 & $0.020^{*}$ & $1.429(1.059,1.929)$ \\
\hline EMVI+/- & 0.871 & $<0.001^{*}$ & $2.389(2.149,2.657)$ & 0.496 & $<0.001^{*}$ & $0.000(0.000,0.000)$ \\
\hline $\mathrm{PNI}+/-$ & 0.810 & $<0.001^{*}$ & $2.249(2.019,2.505)$ & 0.465 & $<0.001^{*}$ & 1.191(1.009,1.407) \\
\hline $\mathrm{CRM}+/-$ & 1.460 & $<0.001^{*}$ & $4.305(3.177,5.832)$ & 0.993 & $<0.001^{*}$ & $1.429(1.059,1.929)$ \\
\hline Epidural anesthesia/ General anesthesia & -0.020 & 0.708 & $0.980(0.883,1.088)$ & & & \\
\hline ASA & & $0.002^{*}$ & & & & \\
\hline II/I & 0.143 & $0.011^{*}$ & $1.153(1.034,1.287)$ & & & \\
\hline III-V/I & 0.448 & $0.002^{*}$ & $1.565(1.178,2.079)$ & & & \\
\hline Haemoglobin $(\mathrm{g} / \mathrm{l})$ & -0.007 & $<0.001^{*}$ & $0.993(0.991,0.995)$ & -0.007 & $<0.001^{*}$ & $0.993(0.990,0.997)$ \\
\hline Albumin $(\mathrm{g} / \mathrm{l})$ & -0.041 & $<0.001^{*}$ & $0.960(0.948,0.973)$ & -0.021 & 0.008 & $0.979(0.964,0.995)$ \\
\hline Operation duration & 0.004 & $<0.001^{*}$ & $1.004(1.003,1.005)$ & 0.005 & $<0.001^{*}$ & $1.005(1.003,1.006)$ \\
\hline Number of lymph nodes examined & -0.037 & $<0.001^{*}$ & $0.964(0.953,0.974)$ & -0.031 & $<0.001^{*}$ & $0.970(0.961,0.979)$ \\
\hline
\end{tabular}




\section{Factors associated with operation duration}

In order to explore the influencing factors of operation duration, we investigted the correlation between operation duration and other clinico-pathological characteristics. Operation methods, surgeons' qualifications, anesthesia methods, histological type, age, gender, BMI and tumor site were associated with operation duration (Table 5). More importantly, surgical approach and surgeons' qualifications had a meaningful correlation coefficient with $\mathrm{r}=0.464$ and 0.450 respectively, which indicated that open surgery and surgery by junior surgeons had longer operation duration.

Table 5. Correlation between characteristics of surgery and duration of surgery

\begin{tabular}{lll}
\hline Characteristics & $\begin{array}{l}\text { Correlation } \\
\text { Coefficient }\end{array}$ & P value \\
\hline Operation methods: laparoscope/open surgery & 0.464 & $<0.001^{*}$ \\
Surgeon's qualification: Young/Old & 0.450 & $<0.001^{*}$ \\
Anesthesia methods: Epidural/ General & 0.028 & $0.029^{*}$ \\
anesthesia & & \\
Histological type & 0.025 & $0.048^{*}$ \\
ASA & -0.003 & 0.789 \\
Staging & -0.017 & 0.180 \\
Number of lymph nodes examined & -0.024 & 0.056 \\
Age & -0.044 & $0.001^{*}$ \\
Female/male & -0.059 & $<0.001^{*}$ \\
BMI & 0.074 & $<0.001^{*}$ \\
Tumor site & -0.063 & $<0.001^{*}$ \\
\hline
\end{tabular}

\section{Discussion}

Our study suggests that longer operation duration is an important independent risk factor for prognosis of patients undergoing colorectal surgery, and increases total costs in hospital, LOS in hospital, LOS in the ICU, and costs associated with of drugs and antibiotics. Concerning long-term prognosis of patients, prolonged operation duration was associated with poorer OS and DFS after surgery. Furthermore, our results revealed that surgical approach and surgeons' qualifications are important factors affecting operation duration, even when the analysis was restricted to surgeons with more than 10 years of surgical experience.

Our results are consistent with previous studies showing that prolonged operation duration was associated with in-hospital costs and the prognosis of patients. Unsurprisingly, prolonged operation duration increased operative field exposure time, which might increase bleeding and stress response. It is well known that systemic inflammatory response can increase LOS and affect the immune system. Importantly, the current study indicated that surgeons' qualifications had a significant effect on operation duration. Therefore, it may be possible to reduce the operation time and thus improve the prognosis of patients by improving the surgical skills of surgeons and strengthening the cooperation of all parties. In addition, surgical approach is also an important factor affecting the operation duration. During the period 2011-2016, laparoscopic colorectal surgery had only recently been introduced at our hospital. Influenced by the learning curve, the duration of endoscopic surgery was significantly longer than that of open surgery. In recent years, with improvements in the level of endoscopic surgery, the duration of endoscopic surgery has decreased significantly, so the advantage of endoscopic surgery is gradually emerging. At present, there is little difference in duration between endoscopic surgery and open surgery. Therefore, the key to reducing operation duration is to improve the surgical skills of the surgeon and strengthen team cooperation.

Previous studies have suggested that prolonged operation duration increases mortality [14, 15], infection risk [7, 13], LOS [10], and the cost of operations [16]. Consistent with these studies, our results also suggest that longer operation duration is related to poor prognosis, including increased costs, increased LOS in hospital and in the ICU, and lower OS and DFS. Additionally, Hernandez et al. found that surgical duration above 120 minutes was associated with increased risk of proximal deep vein thrombosis [17]. However, Wills et al. studied 103044 patients undergoing total hip arthroplasty (THA) from National Surgical Quality Improvement Program (NSQIP) data, and their results agreed with the relationship of prolonged operation duration and superficial surgical site infection, but not deep surgical site infection, organ/space infections, or deep vein thrombosis [18]. Till now, there are only two retrospective studies investigated the impact of prolonged operative time on early postoperative morbidity after colorectal cancer surgery and the conclusions are contradictory. The first study suggested that longer operating times may increase postoperative complication rates for CRC patients with higher BMI [19]. The second study found that there was no relationship between prolonged operation duration and postoperative complications morbidity of patients with rectal cancer undergoing open or robotic surgical resection [9]. With these, we assessed in a large-scale cohort of 6224 patients who underwent radical surgery for colorectal cancer. Our study revealed that prolonged operation duration was not only associated with short-term prognosis but also the long-term prognosis (5-year OS and DFS) of patients.

Most importantly, we pointed out the key factors that affected operation duration. In our research, 
although some factors were statistically associated with operation duration, only surgical approach and surgeons' qualification are meaningful parameters that can be improved. While surgeon experience would affect operation duration, the team familiarity and collaborations could reduce operation duration by a mean of 59 and 22 minutes, respectively [20]. Besides our study, others have suggested that age, gender, tumor site, and anesthesia methods [21] were related to operation duration. Further studies are needed to identify predictive factors for operation duration to guide surgeons in their choice of procedures. It is interesting that several meta-analyses have suggested that robotic operation had longer operation duration than open, laparoscopic, and transanal mesorectal excision [22, 23], but with a shorter hospital stay. So, the mechanism of operation duration on prognosis is complex and warrants further study.

Although the exact mechanisms underlying the positive association between prolonged operation duration and clinical outcomes for CRC patients are not fully understood, plausible links do exist. Possible explanations for the impact of prolonged operation duration on outcomes entail greater surgical trauma, increased risk of venous thromboembolism, augmented exposure to air pathogens, increased suppression of immune function, and decreased nutrient absorption. It is conceivable that longer operative times may also reflect more complex surgical procedures, a wider range of tumors, greater surgical trauma, increased surgical team fatigue, and more technical errors [24, 25]. It is well established that prolonged operation duration can lead to the simultaneous presence of blood stasis, increased coagulation, and endothelial damage, contributing to the risk of venous thromboembolism [25]. Theoretically, longer operative time predisposes the patient to increased exposure to microbes and decreased prophylactic effect of antibiotics, thus leading to subsequent surgical site infections [26]. Finally, it has been shown that longer operative time may result in more delayed postoperative gastrointestinal function recovery, postoperative intestinal obstruction, and decreased absorption of nutrients [27]. Each of these different types of complications could be expected to result in worse postoperative outcomes.

Our research has several limitations. First of all, as a retrospective observational study, the time span of enrollment is wide, and the records of postoperative complications in the medical history of patients are not complete, making it difficult to obtain complete data on postoperative complications which is a good indicator for short-term prognosis.
Additionally, there are many factors that affect the five-year OS and DFS of CRC patients other than the collected variables. The results of the study may be confounded by these uncollected factors. Thus, our conclusions need to be verified by more prospective clinical studies.

\section{Conclusions}

It was found that long operation duration was associated with poor short-term prognosis and long-term mortality. Enhancing surgeons' operative abilities may have a beneficial effect for improving the prognosis of patients. Further studies are needed to confirm the impact of operation duration on patients' prognosis and to explore the mechanisms of the relationship.

\section{Supplementary Material}

Supplementary figures and table. https://www.jcancer.org/v13p1160s1.pdf

\section{Acknowledgments}

The authors thank the participating patients, their families, and staff at the study department.

\section{Funding}

This work was supported by the National Science Foundation of China (81471852) and Shanghai Natural Science Foundation Program

(20ZR1412900).

\section{Competing Interests}

The authors have declared that no competing interest exists.

\section{References}

1. Bray F, Ferlay J, Soerjomataram I, et al. Global cancer statistics 2018: GLOBOCAN estimates of incidence and mortality worldwide for 36 cancers in 185 countries. CA Cancer J Clin. 2018; 68: 394-424.

2. Brenner H, Kloor M, Pox CP. Colorectal cancer. Lancet. 2014; 383: 1490-502.

3. Barresi V, Reggiani Bonetti L, et al. Poorly Differentiated Clusters: Clinical Impact in Colorectal Cancer. Clin Colorectal Cancer. 2017; 16: 9-15.

4. Kim K, Kim YW, Shim H, et al. Differences in clinical features and oncologic. outcomes between metastatic right and left colon cancer. J BUON. 2018; 23: $11-8$.

5. Kim JY, Khavanin N, Rambachan A, et al. Surgical duration and risk of venous thromboembolism. JAMA Surg. 2015; 150: 110-7.

6. Naranje S, Lendway L, Mehle S, et al. Does operative time affect infection rate in primary total. knee arthroplasty? Clin Orthop Relat Res. 2015; 473: 64-9.

7. Samuel AM, Fu MC, Anandasivam NS, et al. After Posterior Fusions for Adult Spinal Deformity, Operative Time is More Predictive of Perioperative Morbidity, Rather Than Surgical Invasiveness: A Need for Speed? Spine. 2017; 42: 1880-7.

8. Daley BJ, Cecil W, Clarke PC, et al. How slow is too slow? Correlation of operative time to. complications: an analysis from the Tennessee Surgical Quality Collaborative. J Am Coll Surg. 2015; 220: 550-8.

9. Duchalais E, Machairas N, Kelley SR, et al. Does prolonged operative time impact. postoperative morbidity in patients undergoing robotic-assisted rectal resection for cancer? Surg Endosc. 2018; 32: 3659-66.

10. Harrison OJ, Smart NJ, White P, et al. Operative time and outcome of enhanced recovery after surgery after laparoscopic colorectal surgery. JSLS. 2014; 18: 265-72.

11. Scheer A, Martel G, Moloo H, et al. Laparoscopic colon surgery: does operative time matter? Dis Colon Rectum. 2009; 52: 1746-52. 
12. Butt ZM, Fazili A, Tan W, et al. Does the presence of significant risk factors affect. perioperative outcomes after robot-assisted radical cystectomy? BJU Int. 2009; 104: 986-90.

13. Wang Q, Goswami $\mathrm{K}$, Shohat $\mathrm{N}$, et al. Longer Operative Time Results in a Higher Rate of. Subsequent Periprosthetic Joint Infection in Patients Undergoing Primary Joint Arthroplasty. J Arthroplasty. 2019; 34: 947-53.

14. Valsangkar N, Salfity HVN, Timsina L, et al. Operative time in esophagectomy: Does it affect. outcomes? Surgery. 2018; 164: 866-71.

15. Inaba CS, Koh CY, Sujatha-Bhaskar S, et al. Operative time as a marker of quality in bariatric surgery. Surg Obes Relat Dis. 2019; 15: 1113-20.

16. Shussman N, Kedar A, Elazary R, et al. Reusable single-port. access device shortens operative time and reduces operative costs. Surg Endosc. 2014; 28: 1902-7.

17. Hernandez AJ, Almeida AM, Fávaro E, et al. The influence of tourniquet use and operative. time on the incidence of deep vein thrombosis in total knee arthroplasty. Clinics. 2012; 67: 1053-7.

18. Wills BW, Sheppard ED, Smith WR, et al. Impact of operative time on early joint infection. and deep vein thrombosis in primary total hip arthroplasty. Orthop Traumatol Surg Res. 2018; 104: 445-8.

19. Hede P, Sörensson MA, Polleryd P, et al. Influence of BMI on short-term surgical outcome after colorectal cancer surgery: a study based on the Swedish national quality registry. Int J Colorectal Dis. 2015; 30: 1201-7.

20. Xu R, Carty MJ, Orgill DP, et al. The teaming curve: a longitudinal study of the influence of. surgical team familiarity on operative time. Ann Surg. 2013; 258: 953-7.

21. Lindeborg MM, Puram SV, Sethi RKV, et al. Predictive factors for prolonged operative time in. head and neck patients undergoing free flap reconstruction. Am J Otolaryngol. 2020; 41: 102392.

22. Simillis C, Lal N, Thoukididou SN, et al. Open Versus Laparoscopic Versus Robotic Versus Transanal Mesorectal Excision for Rectal Cancer: A Systematic Review and Network Meta-analysis. Ann Surg. 2019; 270: 59-68.

23. Braga LH, Pace K, DeMaria J, et al. Systematic review and meta-analysis of robotic-assisted. versus conventional laparoscopic pyeloplasty for patients with ureteropelvic junction obstruction: effect on operative time, length of hospital stay, postoperative complications, and success rate. Eur Urol. 2009; 56: 848-57.

24. Evans C, Lim J, Gatzen C, et al. Factors influencing laparoscopic colorectal operative duration. and its effect on clinical outcome. Surg Laparosc Endosc Percutan Tech. 2012; 22: 437-42.

25. Cheng H, Clymer JW, Po-Han Chen B, et al. Prolonged operative duration is associated with. complications: a systematic review and meta-analysis. J Surg Res. 2018; 229: 134-44.

26. Wang Q, Goswami K, Shohat N, et al. Longer Operative Time Results in a Higher Rate of. Subsequent Periprosthetic Joint Infection in Patients Undergoing Primary Joint Arthroplasty. J Arthroplasty. 2019; 34: 947-53.

27. Zhou Y, Zhang Y, Guo H, et al. Risk Factors Related to Operative Duration and Their. Relationship with Clinical Outcomes in Pediatric Patients Undergoing Roux-en-Y Hepaticojejunostomy. Front Pediatr. 2020; 8: 590420. 